\title{
The clinical and prognostic value of late Gadolinium enhancement imaging in heart failure with mid-range and preserved ejection fraction
}

\author{
Gijs van Woerden ${ }^{1}$ (D) . Dirk J. van Veldhuisen ${ }^{1} \cdot$ Thomas M. Gorter $^{1} \cdot$ Tineke P. Willems $^{2}$. Vanessa P. M. van Empel ${ }^{3}$. \\ Aniek Peters $^{1}$ - Gabija Pundziute ${ }^{1}$. Jeroen W. op den Akker ${ }^{2} \cdot$ Michiel Rienstra $^{1}$ • B. Daan Westenbrink ${ }^{1}$
}

Received: 25 March 2021 / Accepted: 16 July 2021 / Published online: 22 July 2021

(c) The Author(s) 2021

\begin{abstract}
Heart failure (HF) with mid-range or preserved ejection fraction (HFmrEF; HFpEF) is a heterogeneous disorder that could benefit from strategies to identify subpopulations at increased risk. We tested the hypothesis that HFmrEF and HFpEF patients with myocardial scars detected with late gadolinium enhancement (LGE) are at increased risk for all-cause mortality. Symptomatic HF patients with left ventricular ejection fraction (LVEF) $>40 \%$, who underwent cardiac magnetic resonance (CMR) imaging were included. The presence of myocardial LGE lesions was visually assessed. T1 mapping was performed to calculate extracellular volume (ECV). Multivariable logistic regression analyses were used to determine associations between clinical characteristics and LGE. Cox regression analyses were used to assess the association between LGE and allcause mortality. A total of 110 consecutive patients were included (mean age $71 \pm 10$ years, $49 \%$ women, median $\mathrm{N}$-terminal brain natriuretic peptide (NT-proBNP) $1259 \mathrm{pg} / \mathrm{ml}$ ). LGE lesions were detected in 37 (34\%) patients. Previous myocardial infarction and increased LV mass index were strong and independent predictors for the presence of LGE (odds ratio 6.32, 95\% confidence interval (CI) 2.07-19.31, $p=0.001$ and $1.68(1.03-2.73), p=0.04$, respectively). ECV was increased in patients with LGE lesions compared to those without ( 28.6 vs. $26.6 \%, p=0.04)$. The presence of LGE lesions was associated with a fivefold increase in the incidence of all-cause mortality (hazards ratio 5.3, CI 1.5-18.1, $p=0.009$ ), independent of age, sex, New York Heart Association (NYHA) functional class, NT-proBNP, LGE mass and LVEF. Myocardial scarring on CMR is associated with increased mortality in HF patients with LVEF $>40 \%$ and may aid in selecting a subpopulation at increased risk.
\end{abstract}

Keywords $\mathrm{HFpEF} \cdot \mathrm{HFmrEF} \cdot$ Myocardial scar $\cdot$ Cardiac magnetic resonance imaging $\cdot$ Mortality

\section{Introduction}

Heart failure (HF) with mid-range or with preserved ejection fraction (i.e., left ventricular ejection fraction $(\mathrm{LVEF})>40 \%$ ) has a high morbidity and mortality rate and

B. Daan Westenbrink

b.d.westenbrink@umcg.nl

1 Department of Cardiology, University of Groningen, University Medical Centre Groningen, Hanzeplein 1, PO Box 30.001, 9700 RB Groningen, The Netherlands

2 Department of Radiology, University of Groningen, University Medical Centre Groningen, Groningen, The Netherlands

3 Department of Cardiology, University of Maastricht, Medical University Centre Maastricht, Maastricht, The Netherlands there are currently no evidence-based therapies for this condition [1-4]. The lack of therapeutic options for this population is thought to be related to the heterogeneity of the disease [1, 5-7]. Hence, there is an urgent need for identifying more uniform and high-risk subpopulations in HF patients with LVEF $>40 \%$ [6]. Although echocardiography is key for the diagnosis and classification of HF with LVEF $>40 \%$, it offers limited opportunities to establish the underlying aetiology [8].

Cardiac magnetic resonance (CMR) imaging offers unique opportunities for tissue characterisation that may assist in the identification of distinct subpopulations of HF with LVEF $>40 \%$. In particular, the ability of CMR to detect focal myocardial scars using late gadolinium enhancement (LGE) imaging is of interest, as myocardial fibrosis is thought to be a pathophysiological hallmark of HF with 
LVEF $>40 \%$ [9]. LGE lesions are associated with poor prognosis in HF with reduced ejection fraction (HFrEF, LVEF $\leq 40 \%$ ) and the general elderly population without $\mathrm{HF}$, yet surprisingly little is known about the prevalence and prognostic significance of LGE lesions in HF patients with an LVEF > 40\% [10, 11]. We therefore sought to determine the prevalence and prognostic value of myocardial LGE lesions in patients with HFmrEF and HFpEF.

\section{Materials and methods}

\section{Patient population}

Consecutive symptomatic HF patients (New York Heart Association [NYHA] functional class $\geq 2$ ) who had an LVEF $>40 \%$ on echocardiography between November 2012 and December 2019 were studied. Patients were eligible if $\mathrm{N}$-terminal prohormone of brain natriuretic peptide (NTproBNP) was $>125 \mathrm{pg} / \mathrm{ml}$ and echocardiography showed evidence of LV diastolic dysfunction, left atrial dilatation and/or LV hypertrophy, according to current European Society of Cardiology criteria [5]. Patients were excluded from the present analysis if they had LVEF $\leq 40 \%$ on either echocardiography or CMR imaging, (corrected) congenital heart disease, genetically proven hypertrophic-, or dilated cardiomyopathy, or if they had more than moderate leftsided valvular disease. Other exclusion criteria included myocardial infarction $<3$ months prior to CMR imaging contra-indications for CMR imaging, including claustrophobia and presence of pacemaker or internal cardiac defibrillator (ICD). A study flowchart is shown in Fig. 1. Previous myocardial infarction was assessed by reviewing the medical records. All patients were part of a standard diagnostic protocol for HF patients with an LVEF $>40 \%$. This protocol consisted of a thorough examination including laboratory testing, echocardiography and routine cardiac magnetic resonance (CMR) imaging for the aetiology of HF. Serum NT-proBNP and estimated glomerular filtration rate (eGFR) were determined using an immunoassay based on electrochemiluminescence (Elecsys, Roche Diagnostics, Mannheim, Germany). The Institutional Review Board of the University Medical Centre Groningen approved the study and because of the retrospective nature of the study, the need for individual informed consent was waived. The present study was in concordance with the principles outlined in the Declaration of Helsinki.

\section{Echocardiography}

Echocardiographic parameters were assessed according to the current recommendations for cardiac chamber

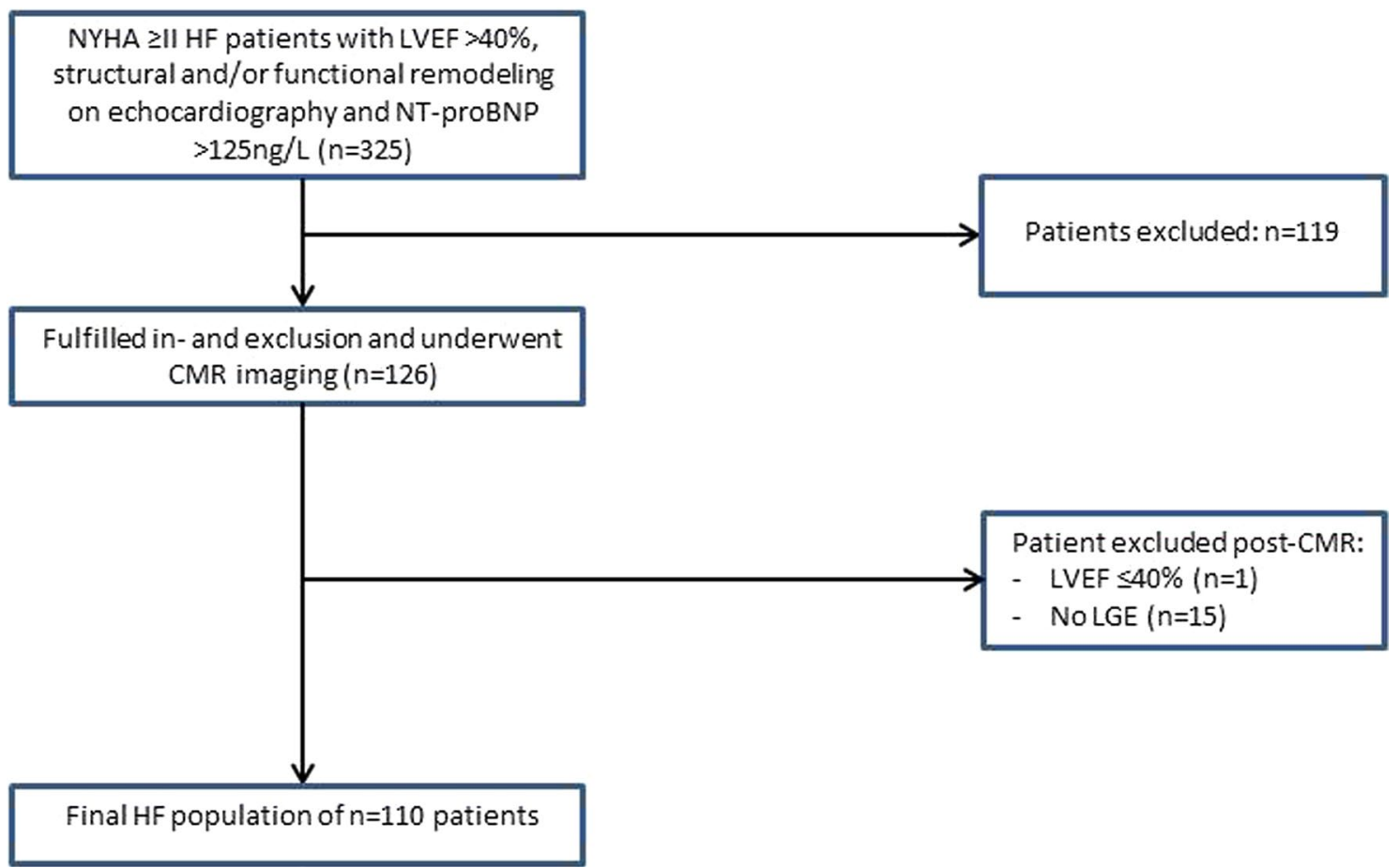

Fig. 1 Study flowchart. NYHA New York Heart Association, $H F$ heart failure, $L V E F$ left ventricular ejection fraction, NT-proBNP $N$-terminal pro brain natriuretic peptide, ICD internal cardiac defi- brillator, $C M R$ cardiac magnetic resonance, $L G E$ late gadolinium enhancement. No $L G E$ no gadolinium administration or insufficient quality 
quantification and included: LV systolic function, LV diastolic function, left atrial volume index, LV mass index, valvular stenosis and/or regurgitation and the peak pressure gradient across the tricuspid valve [12].

\section{Cardiac magnetic resonance imaging}

\section{General assessment}

CMR was performed using a standard protocol for the acquisition of cardiac volumes and functional parameters, as previously published by our group [7]. In brief, all CMR studies were performed using a 1.5 Tesla scanner (Philips, Amsterdam, The Netherlands and Siemens, Erlangen, Germany). ECG-triggered cine loop images were obtained during breath hold at end-expiration, using a retrospectively gated cine steady-state, free-precession sequence. Approximately, 15 short-axis slices from base to apex were obtained. CMR images for LV, right ventricular (RV), left atrial and right atrial volume, function and contractility were analysed off-line by two observers (G.W. and T.M.G.) using dedicated software (QMass 7.6, QStrain 2.0, Medis, Leiden, The Netherlands). The assessment of cardiac volumes, function, and contractility have previously been described by our group [7]. HF patients with LVEF of 40-49\% were classified as HFmrEF, and HF patients with LVEF of $\geq 50 \%$ as HFpEF, according to current ESC criteria [5].

\section{Late gadolinium enhancement}

Using the same slice locations, LGE images were acquired $10 \mathrm{~min}$ after intravenous administration of $0.2 \mathrm{mmol} / \mathrm{kg}$ gadolinium-based contrast agent (Dotarem, Gorinchem, the Netherlands; $0.2 \mathrm{mmol} / \mathrm{kg}$ and Gadovist, Berlin, Germany; $0.2 \mathrm{mmol} / \mathrm{kg}$ ) [13, 14]. Typical sequence parameters were: (Siemens: TE $1.08 \mathrm{~ms}$; TR $700 \mathrm{~ms}$; flip angle $40^{\circ}$; voxel size $2.3 \times 2.3 \times 6.0 \mathrm{~mm}$, Philips: TE $0.93 \mathrm{~ms}$; TR $2.1 \mathrm{~ms}$; flip angle $25^{\circ}$; voxel size $1.6 \times 1.8 \times 8.0 \mathrm{~mm}$.) The presence of LGE was visually evaluated in a blinded fashion by two observers (G.W. and T.M.G) and confirmed by an observer experienced in clinical cardiac magnetic resonance imaging (B.D.W). The full-width half-maximum technique was used to quantify LGE lesions [15].

\section{T1 mapping}

Native and post-contrast T1 measurements were performed using a Modified Look-Locker inversion recovery (MOLLI) sequence (Siemens: TE $1.13 \mathrm{~ms}$; TR $303.92 \mathrm{~ms}$; flip angle $35^{\circ}$; matrix $360 \times 360 \mathrm{~mm}$, voxel size $1.40 \times 1.40 \times 8 \mathrm{~mm}$, Philips: TE $0.93 \mathrm{~ms}$; TR $2.1 \mathrm{~ms}$; flip angle $35^{\circ}$; matrix $300 \times 300 \mathrm{~mm}$, voxel size $2.0 \times 2.0 \times 10 \mathrm{~mm}$.) Native and post-contrast T1 imaging was performed before and at least 10 min after administration of the gadolinium-based contrast agent. T1 measurements were performed on the mid-papillary short-axis slice. For T1 measurements, the endo- and epicardial borders of the LV were manually traced by three observers (G.W., T.M.G. and A.P.) using QMass 7.6 and 8.1 (Medis, Leiden, The Netherlands). When measuring T1 times of the myocardium, extra attention was paid to exclude the blood volume and regions of LGE lesion. Extracellular volume (ECV) fraction was calculated from the nativeand post-contrast $\mathrm{T} 1$ times and blood haematocrit.

\section{Outcome}

The endpoint used in this study was all-cause mortality. Follow-up time was defined as the time from CMR to death. Medical records were reviewed to assess whether the endpoint was met.

\section{Statistical analysis}

Data are presented as numbers and percentages, mean \pm standard deviation or median with interquartile ranges. Differences in categorical variables were analysed using the Chi-squared test or Fisher's exact test, where appropriate. Differences in normally distributed continuous variables were compared using the independent-sample $t$ test or Wilcoxon signed rank test if variables were not normally distributed. Univariable logistic regression was used to determine which covariates were associated with the presence of LGE lesions. To minimize the risk of type II errors, we considered $p<0.1$ as a significant univariable association and these variables were entered into a multivariable backward selection model. First-line interaction between all covariates with $p<0.1$ for the association with the presence of LGE was assessed.

A Kaplan-Meier plot with log rank test was used to display relation between LGE and outcome, which was defined as all-cause mortality. The association between LGE and all-cause mortality was assessed using a univariable Cox proportional hazard regression model. The multivariable Cox regression model was adjusted for age, sex, NYHA functional class, NT-proBNP, LGE mass and LVEF as these variables are strong determinants of outcome in patients with HFpEF [16-18]. The Cox proportional hazards assumption was assessed by visually inspecting the log minus log survival plots over time, which showed proportionality. Statistical analyses were performed using SPSS (Version 23, Chicago, Illinois). Statistical significance was considered achieved at a $p$ value $<0.05$. 


\section{Results}

\section{Clinical characteristics}

The study population consisted of $110 \mathrm{HF}$ patients (Fig. 1). In 15 patients, LGE imaging was either not performed or the scan was of insufficient quality to assess LGE and these patients were excluded. Mean age was $71 \pm 10$ years,
$49 \%$ were female and the median NT-proBNP was $1259 \mathrm{pg} / \mathrm{ml}$ (Table 1). The clinical characteristics stratified by HFpEF or HFmrEF are depicted in Supplementary Table 1. HFpEF patients were more often female (59 vs. $30 \%, p=0.004)$, and more often had a history of hypertension ( 82 vs. $60 \%, p=0.01)$ and less often renal dysfunction (30 vs. $51 \%, p=0.03$ ).
Table 1 Patient characteristics

\begin{tabular}{|c|c|c|c|c|}
\hline & Total $(n=110)$ & LGE- $(n=73)$ & $\mathrm{LGE}+(n=37)$ & $p$ \\
\hline \multicolumn{5}{|l|}{ Demographics } \\
\hline Age (years) & $70.8 \pm 9.8$ & $71.1 \pm 10.0$ & $70.1 \pm 9.4$ & 0.6 \\
\hline $\mathrm{BMI},\left(\mathrm{kg} / \mathrm{m}^{2}\right)$ & $29.5 \pm 5.9$ & $30.0 \pm 6.3$ & $28.5 \pm 4.9$ & 0.2 \\
\hline Male sex, $n(\%)$ & $56(51 \%)$ & $28(38 \%)$ & $28(76 \%)$ & $<0.001$ \\
\hline NYHA class, $n(\%)$ & & & & 0.4 \\
\hline II & $65(59 \%)$ & $41(56 \%)$ & $24(65 \%)$ & \\
\hline III & $45(41 \%)$ & $32(44 \%)$ & $13(35 \%)$ & \\
\hline Systolic blood pressure (mmHg) & $142.3 \pm 20.8$ & $145.2 \pm 18.0$ & $136.1 \pm 25.0$ & 0.1 \\
\hline Diastolic blood pressure $(\mathrm{mmHg})$ & $74.7 \pm 14.5$ & $73.5 \pm 14.4$ & $77.2 \pm 14.5$ & 0.2 \\
\hline Heart rate $(\mathrm{bpm})$ & $71.8 \pm 13.2$ & $72.6 \pm 13.9$ & $70.2 \pm 11.8$ & 0.4 \\
\hline Coronary revascularization $^{\mathrm{a}}(\%)$ & $32(29 \%)$ & $15(21 \%)$ & $17(46 \%)$ & 0.006 \\
\hline \multicolumn{5}{|l|}{ Comorbidities, $n(\%)$} \\
\hline Hypertension & $82(75 \%)$ & $55(75 \%)$ & $27(73 \%)$ & 0.8 \\
\hline Diabetes mellitus & $35(32 \%)$ & $21(29 \%)$ & $14(38 \%)$ & 0.3 \\
\hline Renal dysfunction & $41(37 \%)$ & $28(38 \%)$ & $13(35 \%)$ & 0.7 \\
\hline Myocardial infarction & $26(24 \%)$ & $8(11 \%)$ & $18(49 \%)$ & $<0.001$ \\
\hline Coronary artery disease $^{\mathrm{b}}$ & $37(34 \%)$ & $16(22 \%)$ & $21(57 \%)$ & $<0.001$ \\
\hline Atrial fibrillation & $52(47 \%)$ & $36(49 \%)$ & $16(43 \%)$ & 0.5 \\
\hline \multicolumn{5}{|l|}{ Medications, $n(\%)$} \\
\hline Beta blocker & $98(89 \%)$ & $65(89 \%)$ & $33(89 \%)$ & 1.0 \\
\hline ACEi/ARB & $72(66 \%)$ & $45(62 \%)$ & $27(73 \%)$ & 0.2 \\
\hline Mineral receptor antagonist & $44(40 \%)$ & $31(43 \%)$ & $13(35 \%)$ & 0.5 \\
\hline Diuretic & $99(90 \%)$ & $66(90 \%)$ & $33(89 \%)$ & 0.8 \\
\hline \multicolumn{5}{|l|}{ Laboratory testing } \\
\hline NT-proBNP (pg/ml) & $1259(670-2531)$ & $1202(630-2242)$ & $1726(688-3631)$ & 0.1 \\
\hline TroponinT (ng/L) & $20(13-31)$ & $17(10-27)$ & $27(16-35)$ & 0.007 \\
\hline eGFR (ml/min*1.73m²) & $57(42-77)$ & $57(41-76)$ & $58(44-81)$ & 0.8 \\
\hline \multicolumn{5}{|l|}{ Echocardiography } \\
\hline LV GLS & $12.1 \pm 3.8$ & $12.3 \pm 4.2$ & $11.6 \pm 2.7$ & 0.3 \\
\hline $\mathrm{E} / \mathrm{e}^{\prime}$ & $11(8-15)$ & $11(8-15)$ & $11(9-16)$ & 0.6 \\
\hline $\operatorname{LVMI}\left(\mathrm{g} / \mathrm{m}^{2}\right)$ & $107.1 \pm 40.0$ & $102.8 \pm 36.7$ & $115.2 \pm 45.0$ & 0.1 \\
\hline $\operatorname{LAVI}\left(\mathrm{ml} / \mathrm{m}^{2}\right)$ & $49.6 \pm 22.4$ & $50.5 \pm 23.6$ & $47.8 \pm 20.0$ & 0.6 \\
\hline
\end{tabular}

Statistical significance was considered achieved at a $p$ value $<0.05$

Quantitative data are presented as mean \pm standard deviation or median with interquartile ranges. Qualitative data are presented as $n(\%) . p$ value comparing heart failure (HF) with late gadolinium enhancement (LGE) and HF without LGE. $A C E i$ angiotensin converting enzyme inhibitor, $A R B$ angiotensin II receptor blocker, $B M I$ body mass index, $L V G L S$ left ventricular global longitudinal strain, $L V M I$ left ventricular mass index, $L A V I$ left atrial volume index, NYHA New York Heart Association

${ }^{a}$ Revascularization was defined as follows: underwent percutaneous coronary intervention and/or coronary artery bypass grafting

${ }^{\mathrm{b}}$ Coronary artery disease was defined as follows: history of myocardial infarction, percutaneous intervention and/or coronary artery bypass grafting 


\section{Clinical and CMR characteristics in patients with or without LGE lesions}

LGE lesions were observed in 37 (34\%) patients, of which $22(20 \%)$ were ischemic-, and $15(14 \%)$ were considered to be non-ischemic LGE lesions (Fig. 2). Of the 22 patients
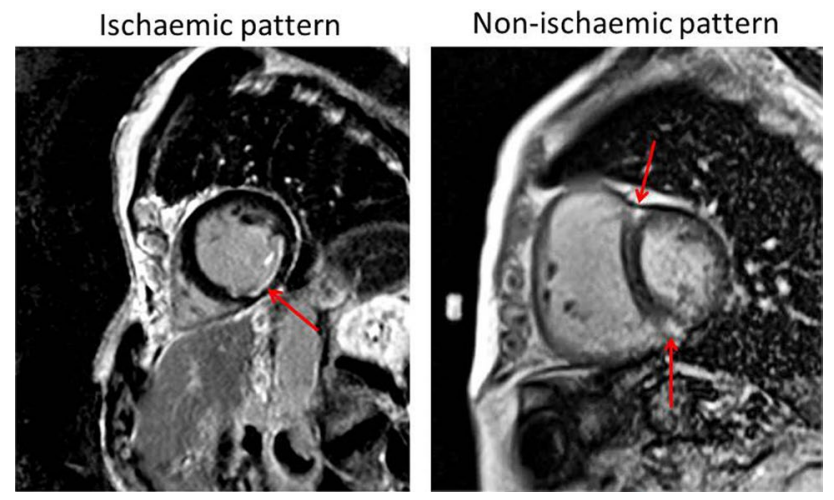

Fig. 2 Examples of late gadolinium enhancement in heart failure patients with left ventricular ejection fraction $>40 \%$ with an ischemic LGE lesion, 8 (36\%) did not have a history of myocardial infarction.

Patients with an LGE lesion were more often male (76 vs. $38 \%, p<0.001)$ and had a previous myocardial infarction more frequently ( 49 vs. $11 \%, p<0.001$ ). In addition, patients with an LGE lesion had increased levels of Troponin $\mathrm{T}$ compared to those without LGE (27 vs. $17 \mathrm{ng} / \mathrm{L}$, $p=0.007)$. The median time interval between troponin $\mathrm{T}$ assessment and CMR imaging was 37 [16-77] days. CMR measurements are depicted in Table 2. LV mass index was almost 20\% higher in patients with LGE lesions compared to patients without LGE $(p=0.02)$. LVEF on echocardiography and CMR were moderately associated $(r=0.38, p<0.001)$. CMR characteristics for HFpEF and HFmrEF are displayed in Supplementary Table 2.

\section{T1 mapping and ECV}

T1 mapping was performed in $75(68 \%)$ patients. In patients with LGE lesions, native T1 times and ECV were significantly increased compared to patients without LGE lesions
Table 2 CMR characteristics: patients with and without late gadolinium enhancement lesions

\begin{tabular}{llll}
\hline & HF LGE- $(n=73)$ & HF LGE $+(n=37)$ & $p$ \\
\hline Structure and function & & & \\
LVESVI $\left(\mathrm{ml} / \mathrm{m}^{2}\right)$ & $41.0 \pm 16.3$ & $47.2 \pm 18.9$ & 0.08 \\
LVEDVI $\left(\mathrm{ml} / \mathrm{m}^{2}\right)$ & $88.1 \pm 24.7$ & $95.4 \pm 25.7$ & 0.2 \\
LVEF $(\%)$ & $54.6 \pm 7.8$ & $51.7 \pm 9.1$ & 0.09 \\
LVMI $\left(\mathrm{g} / \mathrm{m}^{2}\right)$ & $55.2 \pm 19.3$ & $65.3 \pm 25.4$ & $\mathbf{0 . 0 2}$ \\
LV GLS $(\%)$ & $17.7 \pm 5.1$ & $16.8 \pm 4.3$ & 0.4 \\
LV GCS $(\%)$ & $22.8 \pm 6.4$ & $21.0 \pm 6.0$ & 0.2 \\
LV torsion $\left({ }^{\circ}\right)$ & $6.7(2.3-14.1)$ & $8.3(4.3-20.1)$ & 0.07 \\
RVESVI $\left(\mathrm{ml} / \mathrm{m}^{2}\right)$ & $39.4 \pm 14.4$ & $42.4 \pm 21.0$ & 0.4 \\
RVEDVI (ml/m $\left.{ }^{2}\right)$ & $83.9 \pm 21.2$ & $86.2 \pm 25.7$ & 0.6 \\
RVEF $(\%)$ & $53.7 \pm 9.0$ & $52.2 \pm 11.6$ & 0.5 \\
RV GLS $(\%)$ & $20.0 \pm 5.7$ & $20.7 \pm 7.3$ & 0.6 \\
LAESVI $\left(\mathrm{ml} / \mathrm{m}^{2}\right)$ & $60.2 \pm 22.4$ & $60.9 \pm 22.6$ & 0.9 \\
RAESVI $\left(\mathrm{ml} / \mathrm{m}^{2}\right)$ & $46.0 \pm 22.6$ & $45.9 \pm 12.3$ & 1.0 \\
Late gadolinium enhancement & & & \\
LGE, \% of LV mass & & $6.6(4.4-11.2)$ & \\
T1 mapping $(n=75)$ & $1013 \pm 50$ & $1043 \pm 39$ & \\
Native myocardial T1 (ms) & $424 \pm 42$ & $413 \pm 39$ & $28.6 \pm 3.7$ \\
Post-contrast myocardial T1 (ms) & $26.6 \pm 3.3$ & & $\mathbf{0 . 0 1}$ \\
ECV $(\%)$ & & & 0.3 \\
\hline
\end{tabular}

Statistical significance was considered achieved at a $p$ value $<0.05$

Data are presented as mean \pm standard deviation or median with interquartile ranges. $p$ value comparing heart failure (HF) with late gadolinium enhancement (LGE) and HF without LGE. ECV extracellular volume, $L V E F$ left ventricular ejection fraction, $L V E D V I$ left ventricle end-diastolic volume index, $L V E S V I$ left ventricle end-systolic volume index, $L V G L S$ left ventricular global longitudinal strain, $L V G C S$ left ventricular global circumferential strain, $L V M I$ left ventricle mass index, $R V E F$ right ventricular ejection fraction, RVEDVI right ventricle end-diastolic volume index, RVESVI right ventricle end-systolic volume index, $R V G L S$ right ventricular global longitudinal strain, $L A E S V I$ left atrial end-systolic volume index, $R A E S V I$ right atrial end-systolic volume index 
Table 3 Clinical and CMR determinants associated with the presence of late gadolinium enhancement

\begin{tabular}{|c|c|c|c|c|c|c|}
\hline & \multirow[t]{2}{*}{ OR } & \multicolumn{2}{|l|}{ Univariate } & \multirow[b]{2}{*}{ OR } & \multicolumn{2}{|l|}{ Multivariate } \\
\hline & & $95 \% \mathrm{CI}$ & $p$ & & $95 \% \mathrm{CI}$ & $p$ \\
\hline \multicolumn{7}{|l|}{ Demographics } \\
\hline Male sex & 5.00 & $2.06-12.14$ & $<0.001$ & 3.65 & $1.32-10.10$ & 0.01 \\
\hline Systolic blood pressure & 0.98 & $0.96-0.99$ & 0.04 & 0.97 & $0.95-0.99$ & 0.02 \\
\hline Coronary revascularization & 3.29 & $1.39-7.77$ & 0.007 & & & \\
\hline \multicolumn{7}{|l|}{ Comorbidities } \\
\hline Myocardial infarction & 7.70 & $2.90-20.45$ & $<0.001$ & 6.32 & $2.07-19.31$ & 0.001 \\
\hline Coronary artery disease & 4.68 & $1.99-10.99$ & $<0.001$ & & & \\
\hline \multicolumn{7}{|l|}{ Laboratory testing } \\
\hline TroponinT & 2.03 & $1.14-3.64$ & 0.02 & & & \\
\hline \multicolumn{7}{|l|}{ Cardiac magnetic resonance } \\
\hline LVESVI & 1.02 & $0.99-1.04$ & 0.08 & & & \\
\hline LVEF & 0.96 & $0.91-1.00$ & 0.09 & & & \\
\hline $\mathrm{LVMI}^{\mathrm{a}}$ & 1.60 & $1.05-2.44$ & 0.03 & 1.68 & $1.03-2.73$ & 0.04 \\
\hline
\end{tabular}

LVESVI left ventricular end-systolic volume index, $L V E F$ left ventricular ejection fraction, $L V M I$ left ventricle mass index

${ }^{a}$ Per standard deviation increase
(1043 vs. $1013 \mathrm{~ms}, p=0.01$ and 28.6 vs. $26.6 \%, p=0.04$, respectively (Table 2).

\section{Associations between clinical and CMR characteristics and LGE lesions}

Patient- and CMR characteristics with a univariable association $(p<0.1)$ with LGE lesions are depicted in Table 3. Previous myocardial infarction and LV mass index were strong predictors for the presence of LGE lesions and remained independently associated after adjustment (Odds ratio 6.32 with $95 \%$ confidence interval 2.07-19.31, $p=0.001$ and 1.68 (1.03-2.73), $p=0.04$, respectively). Forced entry of age and sex into the model did not improve the predictive accuracy of the model.

\section{Association between myocardial scar and outcome}

During a median follow-up of 34 months (interquartile range 19-53), 19 (17\%) patients died. Of these 19 patients, 11 died due to cardiovascular causes and 8 due to a variety of non-cardiovascular causes such as cancer, and chronic obstructive pulmonary disease. In Fig. 3, a typical CMR example is shown of a patient with a non-ischemic LGE lesion, who died due to cardiovascular causes. Mortality was significantly higher in patients with LGE lesions (Log Rank $p=0.002$, Fig. 4) The presence of LGE was an independent determinant of all-cause mortality after the adjustment for age, sex, NYHA functional class, NT-proBNP, LGE mass and LVEF (adjusted hazards ratio 5.3, 95\% confidence interval 1.5-18.1, $p=0.009$ ) (Table 4). LV diastolic dysfunction,
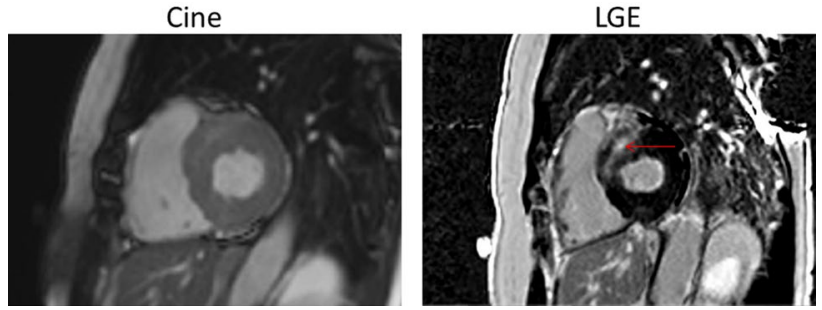

Fig. 3 Typical example of a patient who died due to cardiovascular causes with a late gadolinium enhancement lesion on cardiac magnetic resonance imaging

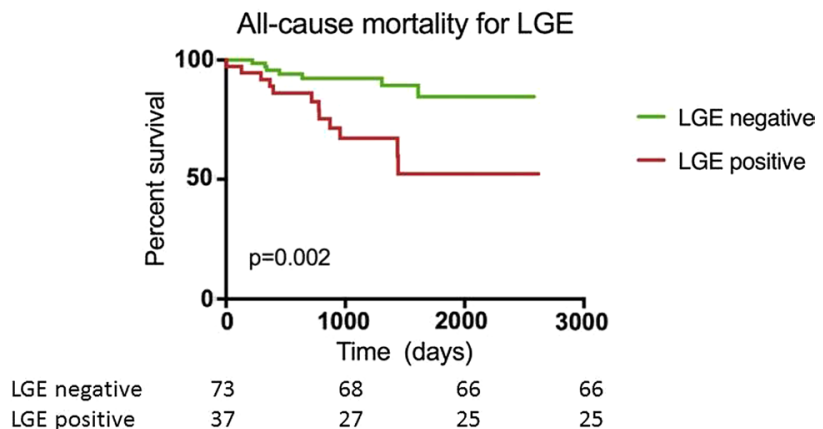

Fig. 4 Kaplan-Meier curves of heart failure patients stratified for the presence of late gadolinium enhancement. $L G E=$ late gadolinium enhancement 
Table 4 Cox regression analysis for all-cause mortality

\begin{tabular}{|c|c|c|c|c|c|c|}
\hline & \multirow[t]{2}{*}{$\mathrm{HR}$} & \multicolumn{2}{|l|}{ Univariate } & \multirow[t]{2}{*}{ HR } & \multicolumn{2}{|c|}{ Multivariate } \\
\hline & & $95 \% \mathrm{CI}$ & $p$ & & $95 \% \mathrm{CI}$ & $p$ \\
\hline LGE presence (yes vs. no) & 3.9 & $1.5-9.9$ & 0.005 & 5.3 & $1.5-18.1$ & 0.009 \\
\hline LGE mass (per 5\%) & 1.00 & $0.96-1.1$ & 0.8 & 0.9 & $0.8-1.1$ & 0.4 \\
\hline Age (per 5 years) & 1.05 & $0.97-1.1$ & 0.3 & 1.08 & $0.97-1.2$ & 0.2 \\
\hline Sex (male vs. female) & 1.5 & $0.6-3.9$ & 0.4 & 1.3 & $0.4-4.4$ & 0.7 \\
\hline NYHA functional class (III vs. II) & 1.9 & $0.7-4.8$ & 0.2 & 1.8 & $0.7-4.9$ & 0.2 \\
\hline NT-proBNP (per doubling) & 1.3 & $0.9-1.8$ & 0.2 & 1.0 & $1.0-1.0$ & 0.6 \\
\hline LVEF (per 5\%) & 1.00 & $0.9-1.1$ & 0.9 & 0.9 & $0.8-1.1$ & 0.9 \\
\hline
\end{tabular}

Statistical significance was considered achieved at a $p$ value $<0.05$

$L G E$ late gadolinium enhancement, NYHA New York Heart Association, LVEF left ventricular ejection fraction, $H R$ hazards ratio, $C I$ confidence interval
LV hypertrophy and left atrial volume index on echocardiography were all not associated with mortality (data not shown).

\section{Discussion}

In the present study, we sought to determine whether CMR with LGE imaging could aid in the identification of a highrisk subgroup of patients with well-defined HFmrEF and HFpEF. Myocardial scarring was present in one third of patients with HFmrEF and HFpEF. In addition, myocardial scars were associated with left ventricular hypertrophy and a history of myocardial infarction. In patients with myocardial scar, ECV values were significantly increased in the myocardium remote from the LGE lesion. Lastly, the presence of myocardial scar was associated with poor outcome. Together, our findings indicate that CMR with LGE imaging may aid in detecting a subpopulation of high-risk $\mathrm{HF}$ patients with LVEF $>40 \%$ and its use should, therefore, be considered in the work-up of HF.

The identification of high-risk subgroups in HF with LVEF $>40 \%$ could be a first step towards better, more tailored treatment of this disease. Our data suggest that the detection of myocardial scar with CMR can help identify such a high-risk subgroup. It is plausible that the difference in survival between patients with- and without an LGE lesion is due to an increase in arrhythmic death. Earlier studies in HFrEF patients showed that the presence of an LGE lesion was associated with ventricular arrhythmic events [19]. However, a recent study by our group showed that sustained- and non-sustained ventricular tachyarrhythmias are uncommon in patients with HFmrEF and HFpEF [20]. Therefore, the association between LGE and ventricular arrhythmias seen in HFrEF, may be different for HFmrEF and HFpEF. In contrast, no distinct subgroups could be made based on cardiac remodelling on echocardiography, further supporting the use of CMR in the work-up of patients with HFmrEF and HFpEF.

Our data support the findings of a prior study, which reported increased mortality and HF hospitalizations in HFpEF patients with myocardial scar on CMR [21]. However, two different studies in HFpEF patients failed to detect a clear association between LGE lesions and outcome [22, 23]. A potential explanation for this discrepancy may be that the follow-up period of the current study is longer than these studies (median of almost 3 years vs. 1 and 2 years). In addition, we also included HF patients with LVEF between 41 and $49 \%$, which is different from previous reports who solely included HF patients with LVEF $>50 \%$.

In our study, LVEF and LV GLS were not associated with LGE or all-cause mortality. This is in line with previous studies, showing that LV systolic function is not independently associated with outcome in patients with HFpEF [24]. LGE volume has been associated with deterioration of LVEF in dilated cardiomyopathy patients in follow-up [25]. Whether this also true for patients with HFmrEF and HFpEF remains to be elucidated.

Native T1 and ECV values were significantly increased in the myocardium remote from the LGE lesion, indicating that patients with a focal myocardial scar also display more interstitial myocardial fibrosis. These findings suggest that focal fibrosis is also associated with more general fibrotic heart disease in HF with LVEF $>40 \%$ and may reflect a higher burden of heart failure.

\section{Study limitations}

Our study is exploratory in nature and suffers from general limitations associated with its retrospective nature. In addition, a few specific limitations should be acknowledged. First, because T1/ECV mapping became available in our center during the course of the study, it is only available in 75 out of 110 patients. Second, although our population 
was meticulously characterised, our sample size is relatively small and our mortality rate was lower than in most HFm$\mathrm{rEF} / \mathrm{HFpEF}$ trials. The incidence and prognostic significance of LGE may, therefore, be different in the general HFmrEF/ HFpEF population. Fourth, we did not include a control group, and we cannot rule out that the association between LGE and mortality also applies to similar subjects without HF.

\section{Clinical implications}

Our study has shown that a considerable amount of HF patients with LVEF $>40 \%$ have myocardial scars and these patients are at increased risk. The presence of an LGE lesion may not only aid in follow-up, but the pattern of LGE may also assist in the diagnosis of the underlying cause of HFpEF/HFmrEF. For instance, of the 22 patients in our study with an ischemic LGE scar, 8 (36\%) did not have a history of myocardial infarction. These findings suggest that HF was caused by a silent myocardial infarction, and these patients may benefit from anti-ischemic therapy. On the other hand, clinical trials may also profit from selecting HF patients with LGE lesions, as this would result in a study population at increased risk.

\section{Conclusions}

Myocardial scarring on CMR is associated with increased mortality in HF patients with LVEF $>40 \%$ and may aid in selecting a subpopulation of patients at increased risk.

Supplementary Information The online version contains supplementary material available at https://doi.org/10.1007/s00380-021-01910-2.

\section{Declarations}

\section{Conflict of interest None.}

Open Access This article is licensed under a Creative Commons Attribution 4.0 International License, which permits use, sharing, adaptation, distribution and reproduction in any medium or format, as long as you give appropriate credit to the original author(s) and the source, provide a link to the Creative Commons licence, and indicate if changes were made. The images or other third party material in this article are included in the article's Creative Commons licence, unless indicated otherwise in a credit line to the material. If material is not included in the article's Creative Commons licence and your intended use is not permitted by statutory regulation or exceeds the permitted use, you will need to obtain permission directly from the copyright holder. To view a copy of this licence, visit http://creativecommons.org/licenses/by/4.0/.

\section{References}

1. Lam CSP, Voors AA, de Boer RA, Solomon SD, van Veldhuisen DJ (2018) Heart failure with preserved ejection fraction: from mechanisms to therapies. Eur Heart J 39:2780-2792

2. van Veldhuisen DJ, Linssen GC, Jaarsma T, van Gilst WH, Hoes AW, Tijssen JG, Paulus WJ, Voors AA, Hillege HL (2013) B-type natriuretic peptide and prognosis in heart failure patients with preserved and reduced ejection fraction. J Am Coll Cardiol 61:1498-1506

3. Suzuki S, Motoki H, Minamisawa M, Okuma Y, Shoin W, Okano T, Kimura K, Ebisawa S, Okada A, Kuwahara K (2019) Prognostic significance of high-sensitivity cardiac troponin in patients with heart failure with preserved ejection fraction. Heart Vessels 34:1650-1656

4. Okuno K, Naito Y, Asakura M, Sugahara M, Ando T, Yasumura S, Nagai T, Saito Y, Yoshikawa T, Masuyama T, Anzai T (2019) Effective blood hemoglobin level to predict prognosis in heart failure with preserved left ventricular ejection fraction: results of the Japanese heart failure syndrome with preserved ejection fraction registry. Heart Vessels 34:1168-1177

5. Ponikowski P, Voors AA, Anker SD, Bueno H, Cleland JG, Coats AJ, Falk V, Gonzalez-Juanatey JR, Harjola VP, Jankowska EA, Jessup M, Linde C, Nihoyannopoulos P, Parissis JT, Pieske B, Riley JP, Rosano GM, Ruilope LM, Ruschitzka F, Rutten FH, van der Meer P, Authors TF, Members DR (2016) 2016 ESC Guidelines for the diagnosis and treatment of acute and chronic heart failure: the Task Force for the diagnosis and treatment of acute and chronic heart failure of the European Society of Cardiology (ESC). Developed with the special contribution of the Heart Failure Association (HFA) of the ESC. Eur J Heart Fail 18:891-975

6. Tromp J, Westenbrink BD, Ouwerkerk W, van Veldhuisen DJ, Samani NJ, Ponikowski P, Metra M, Anker SD, Cleland JG, Dickstein K, Filippatos G, van der Harst P, Lang CC, Ng LL, Zannad F, Zwinderman AH, Hillege HL, van der Meer P, Voors AA (2018) Identifying pathophysiological mechanisms in heart failure with reduced versus preserved ejection fraction. J Am Coll Cardiol 72:1081-1090

7. van Woerden G, Gorter TM, Westenbrink BD, Willems TP, van Veldhuisen DJ, Rienstra M (2018) Epicardial fat in heart failure patients with mid-range and preserved ejection fraction. Eur $\mathbf{J}$ Heart Fail 20:1559-1566

8. Hummel YM, Liu LCY, Lam CSP, Fonseca-Munoz DF, Damman K, Rienstra M, van der Meer P, Rosenkranz S, van Veldhuisen DJ, Voors AA, Hoendermis ES (2017) Echocardiographic estimation of left ventricular and pulmonary pressures in patients with heart failure and preserved ejection fraction: a study utilizing simultaneous echocardiography and invasive measurements. Eur J Heart Fail 19:1651-1660

9. Kasner M, Westermann D, Lopez B, Gaub R, Escher F, Kuhl U, Schultheiss HP, Tschope C (2011) Diastolic tissue Doppler indexes correlate with the degree of collagen expression and cross-linking in heart failure and normal ejection fraction. J Am Coll Cardiol 57:977-985

10. Ganesan AN, Gunton J, Nucifora G, McGavigan AD, Selvanayagam JB (2018) Impact of Late Gadolinium Enhancement on mortality, sudden death and major adverse cardiovascular events in ischemic and nonischemic cardiomyopathy: a systematic review and meta-analysis. Int J Cardiol 254:230-237

11. Shanbhag SM, Greve AM, Aspelund T, Schelbert EB, Cao JJ, Danielsen R, Thornorgeirsson G, Sigurethsson S, Eiriksdottir G, Harris TB, Launer LJ, Guethnason V, Arai AE (2019) Prevalence and prognosis of ischaemic and non-ischaemic myocardial fibrosis in older adults. Eur Heart J 40:529-538 
12. Lang RM, Badano LP, Mor-Avi V, Afilalo J, Armstrong A, Ernande L, Flachskampf FA, Foster E, Goldstein SA, Kuznetsova T, Lancellotti P, Muraru D, Picard MH, Rietzschel ER, Rudski L, Spencer KT, Tsang W, Voigt JU (2015) Recommendations for cardiac chamber quantification by echocardiography in adults: an update from the American Society of Echocardiography and the European Association of Cardiovascular Imaging. J Am Soc Echocardiogr 28:1-39.e14

13. Kramer CM, Barkhausen J, Flamm SD, Kim RJ, Nagel E, Society for Cardiovascular Magnetic Resonance Board of Trustees Task Force on Standardized Protocols (2008) Standardized cardiovascular magnetic resonance imaging (CMR) protocols, society for cardiovascular magnetic resonance: board of trustees task force on standardized protocols. J Cardiovasc Magn Reson 10:35

14. Te Rijdt WP, Ten Sande JN, Gorter TM, van der Zwaag PA, van Rijsingen IA, Boekholdt SM, van Tintelen JP, van Haelst PL, Planken RN, de Boer RA, Suurmeijer AJH, van Veldhuisen DJ, Wilde AAM, Willems TP, van Dessel PFHM, van den Berg MP (2019) Myocardial fibrosis as an early feature in phospholamban p.Arg14del mutation carriers: phenotypic insights from cardiovascular magnetic resonance imaging. Eur Heart J Cardiovasc Imaging 20:92-100

15. Mikami Y, Kolman L, Joncas SX, Stirrat J, Scholl D, Rajchl M, Lydell CP, Weeks SG, Howarth AG, White JA (2014) Accuracy and reproducibility of semi-automated late gadolinium enhancement quantification techniques in patients with hypertrophic cardiomyopathy. J Cardiovasc Magn Reson 16:85

16. Dalos D, Mascherbauer J, Zotter-Tufaro C, Duca F, Kammerlander AA, Aschauer S, Bonderman D (2016) Functional status, pulmonary artery pressure, and clinical outcomes in heart failure with preserved ejection fraction. J Am Coll Cardiol 68:189-199

17. Dewan P, Rorth R, Raparelli V, Campbell RT, Shen L, Jhund PS, Petrie MC, Anand IS, Carson PE, Desai AS, Granger CB, Kober L, Komajda M, McKelvie RS, O’Meara E, Pfeffer MA, Pitt B, Solomon SD, Swedberg K, Zile MR, McMurray JJV (2019) Sex-related differences in heart failure with preserved ejection fraction. Circ Heart Fail 12:e006539

18. Komajda M, Carson PE, Hetzel S, McKelvie R, McMurray J, Ptaszynska A, Zile MR, Demets D, Massie BM (2011) Factors associated with outcome in heart failure with preserved ejection fraction: findings from the Irbesartan in Heart Failure with Preserved Ejection Fraction Study (I-PRESERVE). Circ Heart Fail 4:27-35

19. Scott PA, Rosengarten JA, Curzen NP, Morgan JM (2013) Late gadolinium enhancement cardiac magnetic resonance imaging for the prediction of ventricular tachyarrhythmic events: a metaanalysis. Eur J Heart Fail 15:1019-1027
20. van Veldhuisen DJ, van Woerden G, Gorter TM, van Empel VPM, Manintveld OC, Tieleman RG, Maass AH, Vernooy K, Westenbrink BD, van Gelder IC, Rienstra M (2020) Ventricular tachyarrhythmia detection by implantable loop recording in patients with heart failure and preserved ejection fraction: the VIP-HF study. Eur J Heart Fail 22:1923-1929

21. Kato S, Saito N, Kirigaya H, Gyotoku D, Iinuma N, Kusakawa Y, Iguchi K, Nakachi T, Fukui K, Futaki M, Iwasawa T, Taguri M, Kimura K, Umemura S (2015) Prognostic significance of quantitative assessment of focal myocardial fibrosis in patients with heart failure with preserved ejection fraction. Int J Cardiol 191:314-319

22. Roy C, Slimani A, de Meester C, Amzulescu M, Pasquet A, Vancraeynest D, Beauloye C, Vanoverschelde JL, Gerber BL, Pouleur AC (2018) Associations and prognostic significance of diffuse myocardial fibrosis by cardiovascular magnetic resonance in heart failure with preserved ejection fraction. J Cardiovasc Magn Reson 20:55

23. Schelbert EB, Fridman Y, Wong TC, Abu Daya H, Piehler KM, Kadakkal A, Miller CA, Ugander M, Maanja M, Kellman P, Shah DJ, Abebe KZ, Simon MA, Quarta G, Senni M, Butler J, Diez J, Redfield MM, Gheorghiade M (2017) Temporal relation between myocardial fibrosis and heart failure with preserved ejection fraction: association with baseline disease severity and subsequent outcome. JAMA Cardiol 2:995-1006

24. Kanagala P, Cheng ASH, Singh A, Khan JN, Gulsin GS, Patel P, Gupta P, Arnold JR, Squire IB, Ng LL, McCann GP (2019) Relationship between focal and diffuse fibrosis assessed by CMR and clinical outcomes in heart failure with preserved ejection fraction. JACC Cardiovasc Imaging 12:2291-2301

25. Kubanek M, Sramko M, Maluskova J, Kautznerova D, Weichet J, Lupinek P, Vrbska J, Malek I, Kautzner J (2013) Novel predictors of left ventricular reverse remodeling in individuals with recentonset dilated cardiomyopathy. J Am Coll Cardiol 61:54-63

Publisher's Note Springer Nature remains neutral with regard to jurisdictional claims in published maps and institutional affiliations. 